\title{
THE IMPACT OF ANTHROPOGENIC ACTIVITIES TO THE LEVEL OF SOME POPS RESIDUES IN BOVINE MILK FROM SEVERAL FARMS IN ROMANIA
}

\author{
DIANA IONELA STEGARUS ${ }^{a^{*}}$, ROXANA ELENA IONETE ${ }^{a}$, \\ SIMONA OANCEA ${ }^{b}$, ECATERINA LENGYEL ${ }^{b}$, ADINA FRUM $^{b}$
}

\begin{abstract}
Bovine milk is a product with high consumption rate across the world and therefore its evaluation in terms of consumers' safety and public health is of extreme relevance. In this study, the occurrence of residues of polychlorinated biphenyls (PCBs), organochlorine pesticides and heavy metals was monitored in environmental samples (soil, grassland grass, water) and bovine milk collected from seven locations in Romania, for the assessment of their potential health risk. PCBs were detected in almost all the environmental and milk samples; it highlighted in soil values higher than the normal limit set by the national legislation, but below the alert thresholds and intervention levels. Dichlorodiphenyltrichloroethane (DDT) and its metabolites were found in consistently higher amounts in environmental samples and bovine milk at all sites. Increased values of hexachlorocyclohexane $(\mathrm{HCH})$ were also found in milk samples but without exceeding the maximum admissible levels set by the Romanian legislation. High concentration of cadmium, copper and zinc were found in soil, grass and water samples from the industrial area. Lead exceeded the maximum admissible level in samples from the urban area. The results obtained confirmed the importance of surveilling persistent pollutants in raw milk as part of the healthcare system.
\end{abstract}

Keywords: PCBs, organochlorine pesticides, heavy metals

\section{INTRODUCTION}

The high bioaccumulation potential of persistent organic pollutants (POPs) in environmental matrixes poses serious problems to human health and environment, and therefore an increased concern related to inappropriate

\footnotetext{
a National Research and Development Institute for Cryogenic and Isotopic Technologies ICSI Ramnicu Valcea, 4 Uzinei Street, 240050 Ramnicu Valcea, Romania

b Lucian Blaga University of Sibiu, 10 Victoriei Bvd., 550024 Sibiu, Romania

*Corresponding author: diana.stegarus@icsi.ro
} 
use of pesticides in the agricultural processes is evident [1]. The use of pesticides for crop protection has increased with the rising demand of food quantities on the market due to growing world population and therefore health risk monitoring and assessment measures must be considered. So far, international and regional organizations have set maximum residue limits (MRLs) of different classes and representatives of main food contaminants. Monitoring programs of pesticides, in particular organochlorinated insecticides, have been adopted to ensure food safety, leading also to the enhancement of agricultural resources management and avoidance of economic loss [2,3].

Short-term exposure to pesticides can cause skin and eye irritation, headaches, dizziness, and nausea, and long-term exposure can cause diseases, like: cancer, asthma, diabetes, hormone disruption, cardiovascular diseases, obesity, reproductive and neurological diseases, and learning disabilities. The developing of diseases can start in women embryo. The health risks are difficult to elucidate, because of several factors, like: level of exposure and period of exposure and the type of pesticide, thus the prediction of health hazards is difficult $[4,5]$.

Another group of POPs used in many industrial applications and generally found at low concentrations as contaminant of animal and human food chains, are the polychlorinated biphenyls (PCBs). They decompose very slowly into the environment because of their low solubility in water and high thermal stability [6]. There are 209 congeners of PCBs, numbered from PCB-1 to PCB-209, based on different number and position of chlorine [7], from which six congeners (PCB-28, PCB-52, PCB-101, PCB-138, PCB-153, and PCB-180) occur in high levels in environmental, food and human fluids/tissues samples, being recommended by the Stockholm Convention on Persistent Organic Pollutants (POPs) in 2001 to be monitored. Based on different national recommendations, other congeners may be monitored. The POPs which are banned by Stockholm Convention $(2001,2009)$ exhibit a wide range of toxic effects [8].

A product with high consumption rate is the bovine milk and therefore investigation of its quality in terms of chemical hazard and impact to human health it is of great interest globally. Thus, in order to validate a solid phase extraction method for polychlorinated biphenyl compounds, comparative studies between ocean water and bovine milk were conducted resulting a greater sensibility for the sorbent coating based on polymeric ionic liquids (PIL), at the expense of the PDMS compounds ( $7 \mathrm{~mm}$ polydimethylsiloxane) due to the incorporation of the sorbent particles of the PIL structures. Also, Chen et al. [9] identified 12 polybrominated diphenyl ethers (PBDEs) and 19 polychlorinated biphenyl (PCBs) congeners in milk samples from California, by using rapid methods (QuEChERS, purified by gel permeation chromatography and quantified 
by gas chromatography-mass spectrometry (GC-MS)), concluding that these congeners confirm the previous results of the high levels of PBDEs from California in comparison with other regions of the United States due to the high industrial emissions in the area [9]. In Italy, a series of studies regarding the presence of contamination compounds that can affect human health [10-13] have been performed on cow and buffalo milk samples. The contamination of milk from factories from Brescia (North Italy), was attributed to the contamination of the food of animals. The dioxin-like PCBs were the main contributors to the total levels of OMS-TEQ. They were found in proportions of $87 \%$, in the most contaminated sample. By replacing the contaminated food with one from a non-contaminated region, the levels of OMS-TEQ were dropped up to the ones the European Commission approves of 2 pg OMS-TEQ/g fat [14].

Heavy metals transferred on the chain air-water-soil may pose risk of accumulation in plants through roots, stems or leaves [15-18]. They are also persistent chemicals with toxic effects due to the induced oxidative stress and disturbed metabolic processes, some of them being also carcinogenic agents [19-22].

POPs contamination of soil, water and grassland of agricultural areas can be of great concern for accumulation in the food chain (e.g. dairy and meat products). Therefore, assessing the impact of farms location in terms of the nearness with developed areas (e.g. industrial), but also of the specific agricultural practices used, it is of interest. In this work, the occurrence of residues of some persistent chemicals such as organochlorine pesticides, PCBs and heavy metals was investigated in environmental samples (soil, grass, and water) and bovine milk from farms located in different areas (rural and urban/industrial) in South Romania.

The sampling sites were selected in terms of the anthropogenic activities (e.g. chemical/ industrial companies in the area, and/or intensive agricultural practices), while the POPs were chosen due to their potential risk for health. The PCBs analytes included seven congeners, the PCB-28, PCB-52, PCB-101, PCB-138, PCB-153, PCB-180 and PCB-194; analyzed organochlorine pesticides were lindan $\alpha-, \beta$ - and $\delta$ - isomers of hexachlorocyclohexane $(\mathrm{HCH})$, aldrin, dieldrin, endrin, endosulfan, dichlorodiphenyltrichloroethane (DDT) and its derivatives dichlorodiphenyldichloroethane (DDD), dichlorodiphenyldichloroethylene (DDE); and investigated heavy metals were the lead $(\mathrm{Pb})$, cadmium $(\mathrm{Cd})$, copper (Cu) and zinc (Zn).

The aim of this study is to assess the correlations between the contamination of the environment and bovine milk, regarding the location of farms in terms of nearness with developed areas or specific agricultural practices used. 
DIANA IONELA STEGARUS, ROXANA ELENA IONETE,

SIMONA OANCEA, ECATERINA LENGYEL, ADINA FRUM

\section{RESULTS AND DISCUSSIONS}

\section{Non-dioxin polychlorinated biphenyls in environmental and milk samples}

The concentration of PCBs, pesticides and heavy metals were investigated in environmental samples collected from four counties located in the South of Romania, sites Valcea (VL), Arges (AG), lalomita (IL) and Buzau (BZ). The sampling sites situated around individual farms were as follows: rural (VL-V, VL-E, AG, IL, and BZ), urban-industrial (VL-S near chemical/petrochemical plant) and urban (VL-N resort/thermal springs area/near mountain area). The results on seven investigated polychlorinated biphenyl congeners (PCB28, PCB52, PCB101, PCB138, PCB153, PCB180 and PCB194) are summarized in Figure 1.
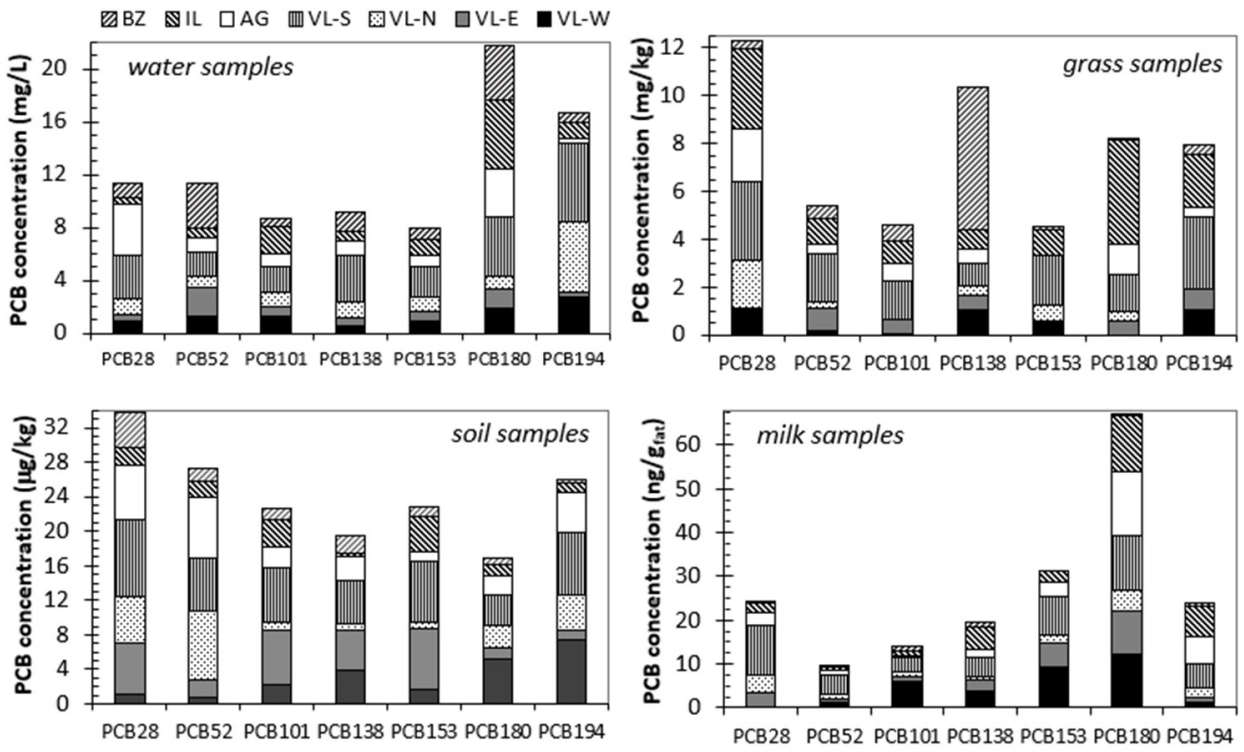

Figure 1. Concentrations of individual PCBs in soil, grass and water samples from the seven sites around the investigated farms and in collected bovine milk samples

The levels of individual PCBs in soil were found higher than normal values $(<0.1 \mu \mathrm{g} / \mathrm{kg}$ for PCB28 and PCB52; and $<0.4 \mu \mathrm{g} / \mathrm{kg}$ for PCB101, PCB 138, PCB 153 and PCB180, respectively) set by Romanian legislation [23]. However, values were situated below the alert thresholds $(2 \mu \mathrm{g} / \mathrm{kg}$ for PCB28 and PCB52; and < $10 \mu \mathrm{g} / \mathrm{kg}$ for PCB101, PCB 138, PCB 153 and PCB180, respectively) for sensitive soils (residential and agriculture areas) 
with the exception of PCB28 in samples from sites VL-Z $(6.070 \mu \mathrm{g} / \mathrm{kg}), \mathrm{VL}-\mathrm{N}$ $(5.240 \mu \mathrm{g} / \mathrm{kg}), A G(6.325 \mu \mathrm{g} / \mathrm{kg})$, and BZ $(4.095 \mu \mathrm{g} / \mathrm{kg})$, and of PCB52 in samples from sites VL-N $(8.030 \mu \mathrm{g} / \mathrm{kg})$ and AG $(7.045 \mu \mathrm{g} / \mathrm{kg})$. All the obtained values were below the intervention levels $(10 \mu \mathrm{g} / \mathrm{kg}$ for PCB28 and PCB52; and $<40 \mu \mathrm{g} / \mathrm{kg}$ for PCB101, PCB 138, PCB 153 and PCB180, respectively). The level of PCBs at VL-S site, an area located near a chemical plant with history in producing pesticides, was the highest found, but below the alert thresholds for less sensitive soils (industrial land use). The sum of PCB congeners ( $\mathrm{PCB} 7$ ) in soil samples from VL-S (Figure 2), which include PCB28, PCB52, PCB101, PCB138, PCB153, and PCB180, was $43.895 \mu \mathrm{g} / \mathrm{kg}$, higher than normal value $(<10 \mu \mathrm{g} / \mathrm{kg})$, but well below the alert thresholds $(250 \mu \mathrm{g} / \mathrm{kg})$ for sensitive soils. The lowest $\Sigma P C B 7$ in soil samples was found in BZ site $(11.385 \mu \mathrm{g} / \mathrm{kg})$. The mean values of soil PCBs including the seven congeners ranged from $1.626 \mu \mathrm{g} / \mathrm{kg}(\mathrm{BZ})$ to $6.271 \mu \mathrm{g} / \mathrm{kg}(\mathrm{VL}-\mathrm{S})$.
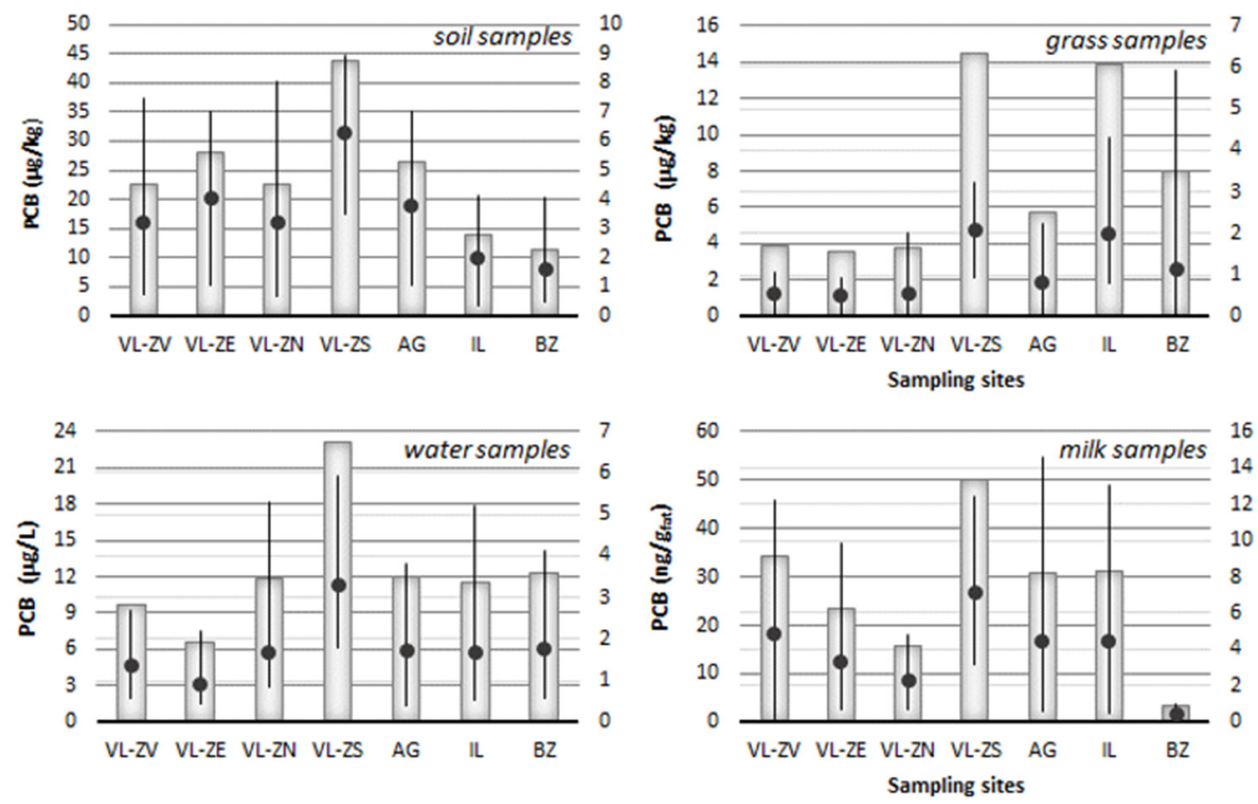

Figure 2. Concentrations of PCBs (sum, min, max and average) in soil, grass and water samples from the seven sites around the investigated farms and in collected bovine milk samples

PCBs were detected in all investigated grass samples, with exception of PCB101 at VL-N site, PCB153 at VL-E and AG sites, PCB180 at VL-W site and PCB194 at VL-N site. Increased levels of PCBs, in particular of high 
DIANA IONELA STEGARUS, ROXANA ELENA IONETE,

SIMONA OANCEA, ECATERINA LENGYEL, ADINA FRUM

chlorinated ones (PCB138, PCB153, PCB180, and PCB194) were found in samples from VL-S (industrial area) and IL sites. The values were below the maximum admissible level imposed for animal feed [24].

Regarding the water samples, the highest mean values of seven PCB congeners and the $\Sigma$ PCB7 were found in VL-S site $(11.820 \mu \mathrm{g} / \mathrm{L})$, while the lowest ones were detected in VL-E site $(6.535 \mu \mathrm{g} / \mathrm{L})$.

PCBs were detected in almost all bovine milk samples. The congener PCB153 was not found in sample BZ. With the exception of sample VL-S, none of the other samples exceeded the maximum level of $40 \mathrm{ng} / \mathrm{g}$ fat as the sum of the indicator of six congeners (PCB28, PCB52, PCB101, PCB138, PCB153 and PCB180) in raw milk as set by EU Regulation [25]. PCB180 showed increased contribution to total PCBs in bovine milk collected from farms at all sites, with exception of BZ site.

\section{Organochlorine pesticides in environmental and bovine milk samples}

Concentration of ten individual organochlorine pesticides $(\alpha-, \beta-, \delta-H C H$, aldrin, dieldrin, endrin, DDE, DDD, DDT and endosulfan) were detected in the investigated environmental samples and bovine milk (Table1).

From the individual pesticides, the DDT and/or its metabolites were found in consistently high amounts in environmental samples and bovine milk at all sites. The highest total concentration of selected pesticides was found in all samples at site VL-S, which is near a chemical industrial area.

However, the sum of DDTs (IDDT) in soil samples was below the Romanian reference values [23], the highest value being of $5.016 \mu \mathrm{g} / \mathrm{kg}$ in VL-S site. Other individual pesticides in higher concentration were the endosulfan (at VL-W and IL sites), aldrin (at BZ), $\mathrm{HCH}$ and endrin (at VL-E). The sum of $\mathrm{HCHs}(\Sigma \mathrm{HCH})$ in soil samples did not exceed the Romanian reference values, except to site VL-S $(7.008 \mu \mathrm{g} / \mathrm{kg})$.

Regarding grass samples, among organochlorine pesticides, the ¿DDTs (Figure 3) were predominant in samples from VL-S site (58.947 $\mu \mathrm{g} / \mathrm{kg}$ ) exceeding the maximum admissible level set by the EU Regulation [26]. Aldrin exceeded normal values in samples from VL-S site (12.079 $\mu \mathrm{g} / \mathrm{kg}$ ). The other samples showed pesticides values below the maximum admissible levels.

Among individual pesticides in water, the $\beta-\mathrm{HCH}, \mathrm{DDE}$ and DDD exceeded normal values $(0.60 \mu \mathrm{g} / \mathrm{L})$ [27] in samples from VL-S, while $\alpha-, \beta$ and $\delta-\mathrm{HCHs}$ were found in high amounts in samples from IL site. 
THE IMPACT OF ANTHROPOGENIC ACTIVITIES TO THE LEVEL OF SOME POPS RESIDUES IN BOVINE MILK FROM SEVERAL FARMS IN ROMANIA

Table 1. Organochlorine pesticides concentration in the environmental samples from the seven sites around the investigated farms and in bovine milk sampled from the selected farms (max values in bold, min values highlighted).

\begin{tabular}{|c|c|c|c|c|c|c|c|}
\hline \multirow{2}{*}{ Pesticides } & \multicolumn{7}{|c|}{ Sampling sites } \\
\hline & VL-W & VL-E & VL-N & VL-S & AG & IL & BZ \\
\hline & \multicolumn{7}{|c|}{ SOIL $(\mu \mathrm{g} / \mathrm{kg})$} \\
\hline \multirow{2}{*}{$\alpha-\mathrm{HCH}$} & 0.051 & 1.026 & 2.003 & 3.002 & 0.037 & 1.122 & 0.654 \\
\hline & \pm 0.0040 & \pm 0.0003 & \pm 0.0004 & \pm 0.0004 & \pm 0.0001 & \pm 0.0009 & \pm 0.0002 \\
\hline \multirow{2}{*}{$\beta-\mathrm{HCH}$} & 0.014 & 0.654 & 0.003 & 4.000 & 0.003 & 0.214 & 0.983 \\
\hline & \pm 0.0000 & \pm 0.0002 & \pm 0.0003 & \pm 0.0002 & \pm 0.0004 & \pm 0.0003 & \pm 0.0004 \\
\hline \multirow{2}{*}{$\delta-\mathrm{HCH}$} & 0.003 & 0.226 & 0.144 & 0.006 & 1.001 & 0.541 & 2.022 \\
\hline & \pm 0.0010 & \pm 0.0001 & \pm 0.0003 & \pm 0.0005 & \pm 0.0005 & \pm 0.0003 & \pm 0.0003 \\
\hline \multirow{2}{*}{ Aldrin } & 0.995 & 0.364 & 1.555 & 0.325 & 0.003 & 0.654 & 1.022 \\
\hline & \pm 0.0070 & \pm 0.0002 & \pm 0.0004 & \pm 0.0004 & \pm 0.0006 & \pm 0.0004 & \pm 0.0001 \\
\hline \multirow{2}{*}{ Dieldrin } & 0.013 & 1.025 & 3.003 & 0.257 & \multirow{2}{*}{ n.d. } & \multirow{2}{*}{ n.d. } & 0.003 \\
\hline & \pm 0.0009 & \pm 0.0003 & \pm 0.0002 & \pm 0.0003 & & & \pm 0.0002 \\
\hline \multirow{2}{*}{ Endrin } & 0.201 & 2.000 & 1.011 & 0.015 & 0.003 & 0.011 & 0.741 \\
\hline & \pm 0.0006 & \pm 0.0006 & \pm 0.0003 & \pm 0.0004 & \pm 0.0004 & \pm 0.0002 & \pm 0.0004 \\
\hline \multirow{2}{*}{ DDE } & 0.039 & 1.003 & 0.642 & 2.004 & 0.007 & 1.363 & 0.856 \\
\hline & \pm 0.0025 & \pm 0.0003 & \pm 0.0003 & \pm 0.0002 & \pm 0.0004 & \pm 0.0005 & \pm 0.0002 \\
\hline \multirow{2}{*}{ DDD } & 1.006 & 0.003 & 0.024 & 1.005 & 0.146 & 2.024 & 0.363 \\
\hline & \pm 0.0008 & \pm 0.0005 & \pm 0.0003 & \pm 0.0001 & \pm 0.0001 & \pm 0.0005 & \pm 0.0005 \\
\hline \multirow{2}{*}{ DDT } & 0.003 & 0.025 & 0.255 & 2.006 & 1.003 & 0.005 & 0.025 \\
\hline & \pm 0.0008 & \pm 0.0007 & \pm 0.0001 & \pm 0.0002 & \pm 0.0003 & \pm 0.0005 & \pm 0.0004 \\
\hline \multirow{3}{*}{ Endosulfan } & 1.037 & 0.666 & 1.000 & 1.003 & 0.364 & 2.051 & 0.644 \\
\hline & \pm 0.0004 & \pm 0.0004 & \pm 0.0004 & \pm 0.0004 & \pm 0.0002 & \pm 0.0004 & \pm 0.0002 \\
\hline & \multicolumn{7}{|c|}{ GRASS $(\mu \mathrm{g} / \mathrm{kg})$} \\
\hline \multirow{2}{*}{$\alpha-\mathrm{HCH}$} & 0.162 & 0.406 & 2.555 & 8.028 & 2.032 & 0.150 & 6.006 \\
\hline & \pm 0.0046 & \pm 0.0449 & \pm 0.0045 & \pm 0.0035 & \pm 0.0007 & \pm 0.0041 & \pm 0.0044 \\
\hline \multirow{2}{*}{$\beta-\mathrm{HCH}$} & 0.844 & 0.511 & 1.027 & 10.521 & 3.023 & 2.318 & 2.024 \\
\hline & \pm 0.0075 & \pm 0.0425 & \pm 0.0050 & \pm 0.0010 & \pm 0.0032 & \pm 0.0458 & \pm 0.0026 \\
\hline \multirow{2}{*}{$\delta-\mathrm{HCH}$} & 1.002 & 2.647 & 1.868 & 16.058 & 6.873 & 14.023 & 2.102 \\
\hline & \pm 0.0180 & \pm 0.0038 & \pm 0.0057 & \pm 0.0043 & \pm 0.0527 & \pm 0.0093 & \pm 0.0004 \\
\hline \multirow{2}{*}{ Aldrin } & 4.026 & 2.131 & 6.739 & 12.079 & 8.075 & 1.307 & 0.377 \\
\hline & \pm 0.0025 & \pm 0.0055 & \pm 0.0040 & \pm 0.0024 & \pm 0.0648 & \pm 0.0539 & \pm 0.0145 \\
\hline \multirow{2}{*}{ Dieldrin } & 5.334 & 7.056 & 5.090 & 7.089 & 11.081 & 4.461 & 0.157 \\
\hline & \pm 0.0006 & \pm 0.0050 & \pm 0.0015 & \pm 0.0003 & \pm 0.0012 & \pm 0.0999 & \pm 0.0054 \\
\hline \multirow{2}{*}{ Endrin } & 8.072 & 5.639 & 7.023 & 25.114 & 6.143 & 4.091 & 8.216 \\
\hline & \pm 0.0497 & \pm 0.0002 & \pm 0.0030 & \pm 0.0006 & \pm 0.0028 & \pm 0.0299 & \pm 0.0346 \\
\hline \multirow{2}{*}{ DDE } & 9.235 & 2.780 & 6.358 & 17.250 & 11.225 & 3.194 & 7.256 \\
\hline & \pm 0.0040 & \pm 0.0024 & \pm 0.0060 & \pm 0.0007 & \pm 0.0050 & \pm 0.1688 & \pm 0.0045 \\
\hline
\end{tabular}


DIANA IONELA STEGARUS, ROXANA ELENA IONETE,

SIMONA OANCEA, ECATERINA LENGYEL, ADINA FRUM

\begin{tabular}{|c|c|c|c|c|c|c|c|}
\hline \multirow{2}{*}{ Pesticides } & \multicolumn{7}{|c|}{ Sampling sites } \\
\hline & VL-W & VL-E & VL-N & VL-S & AG & IL & BZ \\
\hline \multirow{2}{*}{ DDD } & 8.046 & 9.163 & 4.546 & 10.446 & 4.355 & 11.016 & 1.009 \\
\hline & \pm 0.0100 & \pm 0.0015 & \pm 0.0055 & \pm 0.0027 & \pm 0.0048 & \pm 0.0092 & \pm 0.0008 \\
\hline \multirow{2}{*}{ DDT } & 11.151 & 16.091 & 8.869 & 31.251 & 19.571 & 15.062 & 4.488 \\
\hline & \pm 0.1210 & \pm 0.0045 & \pm 0.0045 & \pm 0.0027 & \pm 0.0306 & \pm 0.0394 & \pm 0.0741 \\
\hline \multirow{3}{*}{ Endosulfan } & 2.030 & 6.078 & 12.067 & 17.680 & 10.725 & 5.144 & 16.289 \\
\hline & \pm 0.0055 & \pm 0.0040 & \pm 0.0040 & \pm 0.0002 & \pm 0.6350 & \pm 0.1080 & \pm 0.0320 \\
\hline & \multicolumn{7}{|c|}{ WATER $(\mu \mathrm{g} / \mathrm{L})$} \\
\hline \multirow{2}{*}{$\alpha-\mathrm{HCH}$} & 0.082 & 0.046 & \multirow{2}{*}{ n.d. } & 0.149 & 0.078 & 0.621 & 0.164 \\
\hline & \pm 0.0012 & \pm 0.0020 & & \pm 0.0057 & \pm 0.0034 & \pm 0.0114 & \pm 0.1390 \\
\hline \multirow{2}{*}{$\beta-\mathrm{HCH}$} & 0.003 & 0.007 & 0.016 & 0.917 & 0.059 & 0.742 & 0.191 \\
\hline & \pm 0.0028 & \pm 0.0070 & \pm 0.0018 & \pm 0.0245 & \pm 0.0059 & \pm 0.0008 & \pm 0.0039 \\
\hline \multirow{2}{*}{$\delta-\mathrm{HCH}$} & 0.006 & 0.005 & 0.044 & 0.555 & 0.104 & 0.849 & 0.031 \\
\hline & \pm 0.0011 & \pm 0.0045 & \pm 0.0028 & \pm 0.0049 & \pm 0.0009 & \pm 0.0018 & \pm 0.0002 \\
\hline \multirow{2}{*}{ Aldrin } & \multirow{2}{*}{ n.d. } & 0.082 & \multirow[b]{2}{*}{ n.d. } & 0.004 & 0.028 & 0.364 & \multirow[b]{2}{*}{ n.d. } \\
\hline & & \pm 0.0023 & & \pm 0.0042 & \pm 0.0275 & \pm 0.0020 & \\
\hline \multirow{2}{*}{ Dieldrin } & \multirow[b]{2}{*}{ n.d. } & 0.063 & \multirow[b]{2}{*}{ n.d. } & 0.002 & 0.002 & 0.007 & \multirow[b]{2}{*}{ n.d. } \\
\hline & & \pm 0.0118 & & \pm 0.0025 & \pm 0.0020 & \pm 0.0013 & \\
\hline \multirow{2}{*}{ Endrin } & \multirow{2}{*}{ n.d. } & 0.026 & 0.054 & \multirow[b]{2}{*}{ n.d. } & \multirow[b]{2}{*}{ n.d. } & \multirow[b]{2}{*}{ n.d. } & \multirow[b]{2}{*}{ n.d. } \\
\hline & & \pm 0.0009 & \pm 0.0044 & & & & \\
\hline \multirow{2}{*}{ DDE } & 0.015 & 0.038 & & 0.831 & 0.004 & 0.258 & 0.508 \\
\hline & \pm 0.0011 & \pm 0.0018 & n.a. & \pm 0.0096 & \pm 0.0035 & \pm 0.0031 & \pm 0.1437 \\
\hline & 0.095 & 0.022 & 0.047 & 0.907 & 0.074 & & 0.011 \\
\hline DDD & \pm 0.0012 & \pm 0.0220 & \pm 0.0004 & \pm 0.0038 & \pm 0.0004 & n.a. & \pm 0.0003 \\
\hline דחח & 0.079 & 0.085 & & 0.440 & 0.450 & & 0.449 \\
\hline UDI & \pm 0.0055 & \pm 0.0058 & n.d. & \pm 0.0041 & \pm 0.3708 & n.d. & \pm 0.0018 \\
\hline & 0.028 & & & 0.051 & 0.513 & & 0.035 \\
\hline Endosultan & \pm 0.0280 & n.a. & n.d. & \pm 0.0074 & \pm 0.0035 & n.d. & \pm 0.0008 \\
\hline & & & BOVII & MILK (ng & & & \\
\hline$\alpha-\mathrm{HCH}$ & 3.958 & & & 6.080 & 4.031 & 0.004 & 1.360 \\
\hline a-HCH & \pm 0.0632 & n.d. & n.d. & \pm 0.0018 & \pm 0.3331 & \pm 0.0042 & \pm 0.0973 \\
\hline & 2.065 & 3.254 & 4.266 & 4.812 & 7.046 & 1.257 & 3.031 \\
\hline 及-HCH & \pm 0.0040 & \pm 0.0039 & \pm 0.0050 & \pm 0.4419 & \pm 0.0048 & \pm 0.0049 & \pm 0.0056 \\
\hline$\delta \mathrm{HCH}$ & 2.225 & 8.255 & 3.077 & 9.261 & 3.333 & 0.981 & 0.768 \\
\hline O-HCH & \pm 0.0047 & \pm 0.0050 & \pm 0.0048 & \pm 0.0107 & \pm 0.0019 & \pm 0.0064 & \pm 0.0729 \\
\hline Aldrin & 3.123 & & 2.226 & & 1.262 & 2.353 & \\
\hline Aldrin & \pm 0.0273 & n.d. & \pm 0.0057 & n.d. & \pm 0.0027 & \pm 0.0111 & n.d. \\
\hline Dieldrin & & & 4.256 & 4.149 & 2.085 & 2.118 & 0.355 \\
\hline Dieların & n.d. & n.d. & \pm 0.0048 & \pm 0.0017 & \pm 0.0051 & \pm 0.0235 & \pm 0.3030 \\
\hline Endrin & n.d. & 2.116 & n.d. & n.d. & 1.556 & n.d. & n.d. \\
\hline
\end{tabular}


THE IMPACT OF ANTHROPOGENIC ACTIVITIES TO THE LEVEL OF SOME POPS RESIDUES IN BOVINE MILK FROM SEVERAL FARMS IN ROMANIA

\begin{tabular}{|c|c|c|c|c|c|c|c|}
\hline \multirow{2}{*}{ Pesticides } & \multicolumn{7}{|c|}{ Sampling sites } \\
\hline & VL-W & VL-E & VL-N & VL-S & AG & IL & BZ \\
\hline & & \pm 0.0046 & & & \pm 0.0047 & & \\
\hline \multirow{2}{*}{$p, p^{\prime}-\mathrm{DDE}$} & 6.618 & 5.225 & 3.331 & 6.216 & 4.256 & \multirow[b]{2}{*}{ n.d. } & 1.358 \\
\hline & \pm 0.0233 & \pm 0.0050 & \pm 0.0065 & \pm 0.0048 & \pm 0.0056 & & \pm 0.0035 \\
\hline \multirow{2}{*}{ p,p'-TDE } & 5.259 & 6.386 & 6.581 & 6.084 & 6.576 & 0.707 & 0.958 \\
\hline & \pm 0.0021 & \pm 0.0043 & \pm 0.0073 & \pm 0.0016 & \pm 0.0052 & \pm 0.0674 & \pm 0.0037 \\
\hline \multirow{2}{*}{ DDT } & 7.065 & 6.181 & 7.147 & 9.546 & 7.056 & 6.377 & 2.327 \\
\hline & \pm 0.0038 & \pm 0.0014 & \pm 0.0034 & \pm 0.0046 & \pm 0.0042 & \pm 0.0129 & \pm 0.0047 \\
\hline \multirow{2}{*}{ Endosulfan } & 3.380 & 2.359 & \multirow{2}{*}{ n.d. } & 4.503 & 2.562 & 2.019 & 0.306 \\
\hline & \pm 0.0008 & \pm 0.0020 & & \pm 0.0071 & \pm 0.0025 & \pm 0.0026 & \pm 0.0151 \\
\hline
\end{tabular}

Mean value $(n=3) \pm$ standard deviation

Concerning raw milk samples, the DDT was found in all samples. The highest amount of $\Sigma$ DDTs was registered in sample from VL-S, of 21.846 $\mathrm{ng} / \mathrm{g}_{\mathrm{fat}}$, followed by VL-W (18.942 $\left.\mathrm{ng} / \mathrm{g}_{\mathrm{fat}}\right)$, while the lowest was found in BZ $\left(4.642 \mathrm{ng} / \mathrm{g}_{\mathrm{fat}}\right)$ and IL $\left(7.084 \mathrm{ng} / \mathrm{g}_{\mathrm{fat}}\right)$. The obtained values did not exceed the maximum admissible levels set by Romanian legislation [28].
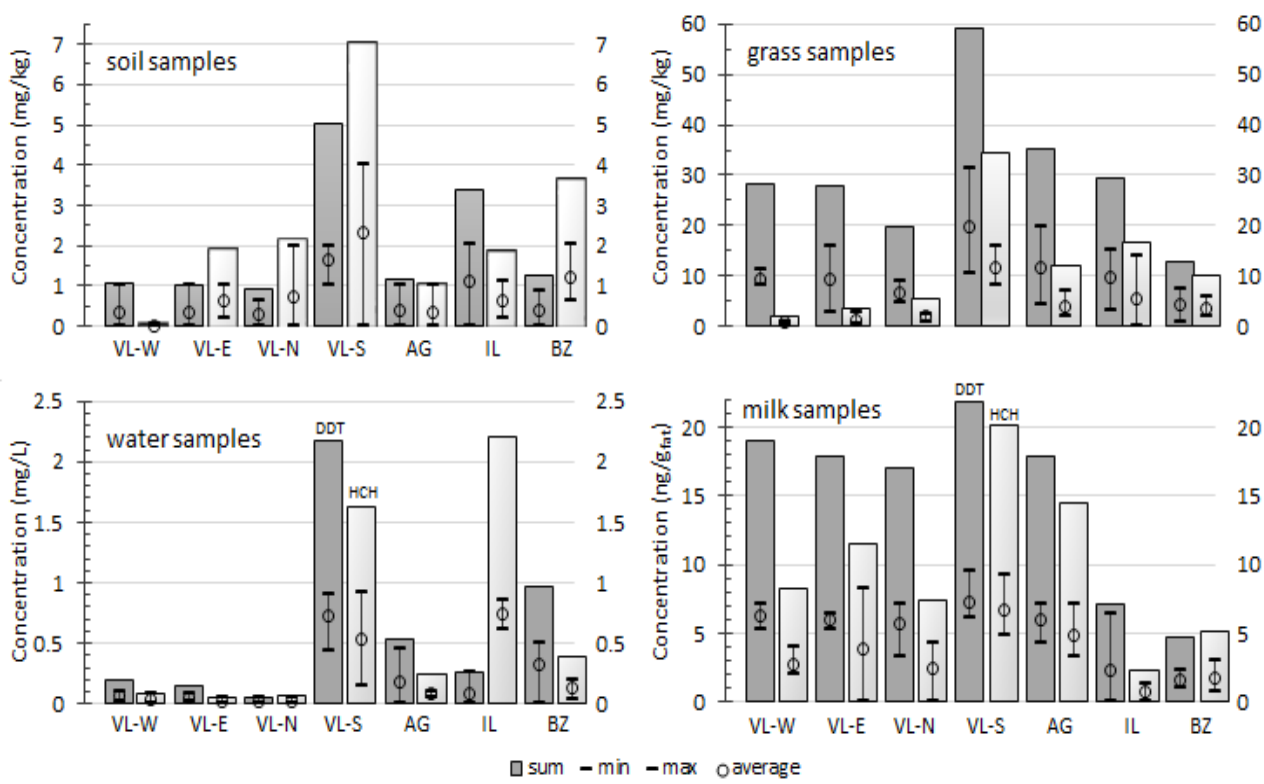

Figure 3. Concentrations of $\Sigma D D T s$ (sum, min, max and average) in soil, grass and water samples from the seven sites around the investigated farms and in collected bovine milk samples 
Our study revealed low levels of pesticides compared with other studies. A study provided by Deti et al. [29] on bovine milk from different regions of Ethiopia, empasized larger quantities of organochlorine pesticides than the ones found in this study, like: DDE, that reaches quantities of up to $91.9 \mathrm{ng} / \mathrm{g}$ fat and EDDT up to $1230 \mathrm{ng} / \mathrm{g}$ fat. The endosulfan was not detected in most of the samples, but in the samples that was detected it reached a quantum of up to $142.1 \mathrm{ng} / \mathrm{g}$ fat and aldrin was found in only one sample and it had a quantity of $11.6 \mathrm{ng} / \mathrm{g}$ fat.

However, increased values of $\alpha-, \beta-$, and $\delta-\mathrm{HCH}$ were found in milk sample from VL-S, $\beta$-, and $\delta-H C H$ in sample from VL-E and $\beta-H C H$ to AG site. Endrin was absent in most of samples, except for VL-E and AG sites.

\section{Heavy metals in environmental}

Regarding the level of heavy metals ( $\mathrm{Pb}, \mathrm{Cd}, \mathrm{Cu}$ and $\mathrm{Zn})$, the obtained results are presented in Table 2. Heavy metals were detected in environmental samples in various concentrations, as follows:

(i) Lead level varied in the range of $7.285 \div 321.110 \mu \mathrm{g} / \mathrm{kg}$ in soil samples (the highest concentration in sample from VL-N, exceeding the maximum admissible level [30], $0 \div 2.505 \mu \mathrm{g} / \mathrm{kg}$ in grass samples (highest value in VL-S) and $0.185 \div 8.020 \mu \mathrm{g} / \mathrm{L}$ in water sample (highest value in VL-S);

(ii) Cadmium level varied in the range of $1.220 \div 9.965 \mu \mathrm{g} / \mathrm{kg}$ in soil samples (the highest concentration to IL), $0.025 \div 1.905 \mu \mathrm{g} / \mathrm{kg}$ in grass samples (highest value in VL-S) and $0.265 \div 2.235 \mu \mathrm{g} / \mathrm{L}$ in water sample (highest value in IL)

(iii) Copper level varied in the range of $46.195 \div 226.725 \mu \mathrm{g} / \mathrm{kg}$ in soil samples (the highest concentration in sample VL-S), $1.430 \div 16.070$ $\mu \mathrm{g} / \mathrm{kg}$ in grass samples (highest value in VL-S) and $4.185 \div 69.030$ $\mu \mathrm{g} / \mathrm{L}$ in water sample (highest value to VL-S)

(iv) Zinc level varied in the range of $88.600 \div 469.601 \mu \mathrm{g} / \mathrm{kg}$ in soil samples (the highest concentration in sample VL-S), $11.575 \div 67.110$ $\mu \mathrm{g} / \mathrm{kg}$ in grass samples (highest value in VL-S) and $539.185 \div$ $2306.090 \mu \mathrm{g} / \mathrm{L}$ in water sample (highest value in VL-ZE)

Soil, grass and water samples at VL-ZS showed high concentration of cadmium, copper and zinc.

Statistical analysis showed extremely significant positive Pearson's correlation coefficients $(p<0.01)$ between cadmium-contaminated soil and cadmium-contaminated water $\left(R^{2}=0.9765\right)$, and between lead-contaminated water and copper-contaminated water $\left(R^{2}=0.9093\right)$, respectively. Copper residues in water high significantly correlated with those in soil $\left(R^{2}=0.7976\right)$ and in grass, respectively $\left(R^{2}=0.8702\right)$. 
THE IMPACT OF ANTHROPOGENIC ACTIVITIES TO THE LEVEL OF SOME POPS RESIDUES IN BOVINE MILK FROM SEVERAL FARMS IN ROMANIA

Table 2. Concentration of $\mathrm{Fe}, \mathrm{Cd}, \mathrm{Cu}$ and $\mathrm{Zn}$ in the environmental samples (soil, grass and water) from the seven sites around the investigated farms

\begin{tabular}{|c|c|c|c|c|c|c|c|}
\hline \multirow{2}{*}{ Samples } & \multicolumn{7}{|c|}{ Sampling sites } \\
\hline & VL-ZV & VL-ZE & VL-ZN & VL-ZS & VL-Z S-E & IL & BZ \\
\hline & \multicolumn{7}{|c|}{ Lead $(\mu \mathrm{g} / \mathrm{kg})$} \\
\hline soil & $\begin{array}{r}9.170 \\
\pm 0.190\end{array}$ & $\begin{array}{r}136.190 \\
\pm 0.060\end{array}$ & $\begin{array}{r}321.110 \\
\pm 0.040\end{array}$ & $\begin{array}{r}47.090 \\
\pm 0.030 \\
\end{array}$ & $\begin{array}{r}156.225 \\
\pm 0.035\end{array}$ & $\begin{array}{r}7.285 \\
\pm 0.035\end{array}$ & $\begin{array}{r}215.135 \\
\pm 0.165\end{array}$ \\
\hline grass & $\begin{array}{r}1.085 \\
\pm 0.065 \\
\end{array}$ & $\begin{array}{r}0.745 \\
\pm 0.115 \\
\end{array}$ & $\begin{array}{r}0.910 \\
\pm 0.060 \\
\end{array}$ & $\begin{array}{r}2.505 \\
+0.145 \\
\end{array}$ & $\begin{array}{r}0.125 \\
\pm 0.125 \\
\end{array}$ & & $\begin{array}{r}0.520 \\
\pm 0.030 \\
\end{array}$ \\
\hline \multirow[t]{2}{*}{ water } & $\begin{array}{r}0.185 \\
\pm 0.015 \\
\end{array}$ & $\begin{array}{r}0.220 \\
\pm 0.040 \\
\end{array}$ & $\begin{array}{r}4.900 \\
\pm 0.130 \\
\end{array}$ & $\begin{array}{r}7.085 \\
+0.065 \\
\end{array}$ & $\begin{array}{r}1.225 \\
\pm 0.135 \\
\end{array}$ & $\begin{array}{r}8.020 \\
\pm 0.130 \\
\end{array}$ & $\begin{array}{r}0.370 \\
+0.030 \\
\end{array}$ \\
\hline & \multicolumn{7}{|c|}{ Cadmium $(\mu \mathrm{g} / \mathrm{kg})$} \\
\hline soil & $\begin{array}{r}1.220 \\
\pm 0.030 \\
\end{array}$ & $\begin{array}{r}5.320 \\
\pm 0.040 \\
\end{array}$ & $\begin{array}{r}4.150 \\
\pm 0.060 \\
\end{array}$ & $\begin{array}{r}9.215 \\
\pm 0.055 \\
\end{array}$ & $\begin{array}{r}2.580 \\
\pm 0.030 \\
\end{array}$ & $\begin{array}{r}9.965 \\
\pm 0.085 \\
\end{array}$ & $\begin{array}{r}6.460 \\
\pm 0.21 \\
\end{array}$ \\
\hline grass & $\begin{array}{r}0.030 \\
\pm 0.030 \\
\end{array}$ & $\begin{array}{r}0.025 \\
\pm 0.025 \\
\end{array}$ & $\begin{array}{r}0.790 \\
\pm 0.050 \\
\end{array}$ & $\begin{array}{r}1.905 \\
\pm 0.035 \\
\end{array}$ & $\begin{array}{r}0.135 \\
\pm 0.035 \\
\end{array}$ & $\begin{array}{r}0.050 \\
+0.000 \\
\end{array}$ & $\begin{array}{r}0.630 \\
\pm 0.060 \\
\end{array}$ \\
\hline water & $\begin{array}{r}0.265 \\
\pm 0.045 \\
\end{array}$ & $\begin{array}{r}0.745 \\
\pm 0.095 \\
\end{array}$ & $\begin{array}{r}0.685 \\
\pm 0.055 \\
\end{array}$ & $\begin{array}{r}1.770 \\
\pm 0.250 \\
\end{array}$ & $\begin{array}{r}0.385 \\
\pm 0.025 \\
\end{array}$ & $\begin{array}{r}2.235 \\
\pm 0.105 \\
\end{array}$ & $\begin{array}{r}1.305 \\
\pm 0.055 \\
\end{array}$ \\
\hline & \multicolumn{7}{|c|}{ Copper $(\mu \mathrm{g} / \mathrm{kg})$} \\
\hline soil & $\begin{array}{r}46.195 \\
\pm 0.055 \\
\end{array}$ & $\begin{array}{r}63.28 \\
\pm 0.030 \\
\end{array}$ & $\begin{array}{r}113.085 \\
\pm 0.055 \\
\end{array}$ & $\begin{array}{r}226.725 \\
\pm 1.445 \\
\end{array}$ & $\begin{array}{r}98.225 \\
\pm 0.055 \\
\end{array}$ & $\begin{array}{r}97.620 \\
\pm 0.470 \\
\end{array}$ & $\begin{array}{r}139.750 \\
\pm 0.310 \\
\end{array}$ \\
\hline grass & $\begin{array}{r}5.190 \\
\pm 0.170 \\
\end{array}$ & $\begin{array}{r}7.320 \\
\pm 0.260 \\
\end{array}$ & $\begin{array}{r}10.450 \\
\pm 0.070 \\
\end{array}$ & $\begin{array}{r}16.070 \\
\pm 0.180 \\
\end{array}$ & $\begin{array}{r}1.430 \\
\pm 0.110 \\
\end{array}$ & $\begin{array}{r}14.065 \\
\pm 0.185 \\
\end{array}$ & $\begin{array}{r}2.705 \\
\pm 0.345 \\
\end{array}$ \\
\hline water & $\begin{array}{r}12.165 \\
\pm 0.195 \\
\end{array}$ & $\begin{array}{r}4.185 \\
\pm 0.335 \\
\end{array}$ & $\begin{array}{r}36.300 \\
\pm 0.250 \\
\end{array}$ & $\begin{array}{r}69.030 \\
\pm 0.120 \\
\end{array}$ & $\begin{array}{r}12.880 \\
\pm 0.140 \\
\end{array}$ & $\begin{array}{r}44.015 \\
\pm 0.075 \\
\end{array}$ & $\begin{array}{r}15.605 \\
\pm 0.455 \\
\end{array}$ \\
\hline & \multicolumn{7}{|c|}{ Zinc $(\mu \mathrm{g} / \mathrm{kg})$} \\
\hline soil & $\begin{array}{r}88.600 \\
\pm 0.030 \\
\end{array}$ & $\begin{array}{r}365.200 \\
\pm 0.320 \\
\end{array}$ & $\begin{array}{r}118.380 \\
\pm 0.040 \\
\end{array}$ & $\begin{array}{r}469.601 \\
\pm 0.030 \\
\end{array}$ & $\begin{array}{r}105.280 \\
\pm 0.030 \\
\end{array}$ & $\begin{array}{r}236.080 \\
\pm 0.07 \\
\end{array}$ & $\begin{array}{r}364.180 \\
\pm 0.070 \\
\end{array}$ \\
\hline grass & $\begin{array}{r}17.225 \\
\pm 0.135 \\
\end{array}$ & $\begin{array}{r}11.575 \\
\pm 0.065 \\
\end{array}$ & $\begin{array}{r}44.785 \\
\pm 0.235 \\
\end{array}$ & $\begin{array}{r}67.110 \\
\pm 0.140 \\
\end{array}$ & $\begin{array}{r}25.160 \\
\pm 0.080 \\
\end{array}$ & $\begin{array}{r}28.945 \\
\pm 0.205 \\
\end{array}$ & $\begin{array}{r}18.140 \\
\pm 0.080 \\
\end{array}$ \\
\hline water & $\begin{array}{r}1216.065 \\
\pm 6.955 \\
\end{array}$ & $\begin{array}{r}2306.090 \\
\pm 9.060 \\
\end{array}$ & $\begin{array}{r}1718.100 \\
\pm 7.040 \\
\end{array}$ & $\begin{array}{r}1107.050 \\
\pm 5.310 \\
\end{array}$ & $\begin{array}{r}2230.930 \\
\pm 25.190 \\
\end{array}$ & $\begin{array}{r}539.185 \\
+438.935 \\
\end{array}$ & $\begin{array}{r}2207.600 \\
\pm 6.430 \\
\end{array}$ \\
\hline
\end{tabular}

Mean value $(n=3) \pm$ standard deviation

A significant correlation $(\mathrm{p}<0.05)$ of PCBs in milk and PCBs in soil $\left(R^{2}=0.7548\right)$ was observed. The correlation analysis of milk samples also indicated a good correlation of PCBs and pesticides $\left(R^{2}=0.7568\right)$.

Regarding the residues in water samples, there was a highly significant correlation $(p<0.05)$ between PCBs and pesticide $\left(R^{2}=0.8068\right)$, cadmium and pesticides $\left(R^{2}=0.8267\right)$, and copper and pesticides $\left(R^{2}=0.8038\right)$. Concerning grass samples, a very significant $(p<0.05)$ positive correlation was found between cadmium and pesticides $\left(R^{2}=0.8157\right)$, and zinc and pesticides $\left(R^{2}=\right.$ $0.8344)$. 
DIANA IONELA STEGARUS, ROXANA ELENA IONETE,

SIMONA OANCEA, ECATERINA LENGYEL, ADINA FRUM

\section{CONCLUSIONS}

Residues of polychlorinated biphenyls, organochlorine pesticides and heavy metals from environment may enter the food webs and generate negative effects in the human body. Contaminated soil, water and grassland grass with organochlorine pesticides, PCBs and heavy metals in areas of farming may be of great concern of accumulation in raw bovine milk.

The results of the present investigation on levels of persistent chemicals (non-dioxin like PCBs, organochlorine pesticides and heavy metals) were determined for environmental and bovine milk samples of local origin. Sampling sites were selected based on historical contamination due to the presence of large chemical / industrial companies and /or intensive agricultural practices.

Samples from sites near industrial areas show relative high contamination. In particular, high chlorinated PCBs (PCB138, PCB153, PCB180, and PCB194), high $\Sigma D D T, \Sigma H C H$, cadmium, copper and zinc were detected in those areas. The analyzed samples did not exceed the maximum admissible levels set by the Romanian legislation for each contaminant, except for the site situated in the industrial are.

The results of this work confirmed the importance of surveilling POPs on the milk chain, from farm to fork, and are important for the public awareness regarding chemical hazards. Therefore, advanced strategy for monitoring milk safety should be applied to identify pathways for contamination.

\section{EXPERIMENTAL SECTION}

\section{Sample collection and storage}

Environmental samples (soil, grass and water) were collected in 2017 from four South Romanian counties, namely: Valcea (VL), Arges (AG), lalomita (IL), and Buzau (BZ), at different sites around local farms, which regularly provide bovine milk for automatic milk dispensers (Figure 4). The sampling sites are listed in Table 3.

From each site (Table 3), approximately $1 \mathrm{~kg}$ of soil samples $(7 \mathrm{~cm}$ deep) were collected from three sampling points situated near the pastures for animals, using a stainless-steel soil sampling probe with a conically-tapered tip for an easier drive-in sample extraction, and stored in zip lock bags to be investigated later in the laboratory. Also, approximately $1 \mathrm{~kg}$ of grass samples from pastures and $1 \mathrm{~L}$ of water were collected and kept at $+4^{\circ} \mathrm{C}$ before analysis. Additionally, fresh bovine milk was sampled from the collecting centers in the investigated areas to assess the potentially residues of PCBs, pesticides and heavy metals. The selection of milk sample supplying sites was based on both economic and consumer safety criteria since the farms/collecting centers 
THE IMPACT OF ANTHROPOGENIC ACTIVITIES TO THE LEVEL OF SOME POPS RESIDUES IN BOVINE MILK FROM SEVERAL FARMS IN ROMANIA

provide important amounts of raw milk to farmers markets vending machines. Milk samples ( $2 \mathrm{~L}$ each) were collected in plastic bottles and transported to the laboratory in cooling boxes with ice packs where they were immediately stored in a freezer at $-24^{\circ} \mathrm{C}$ until analyzed.
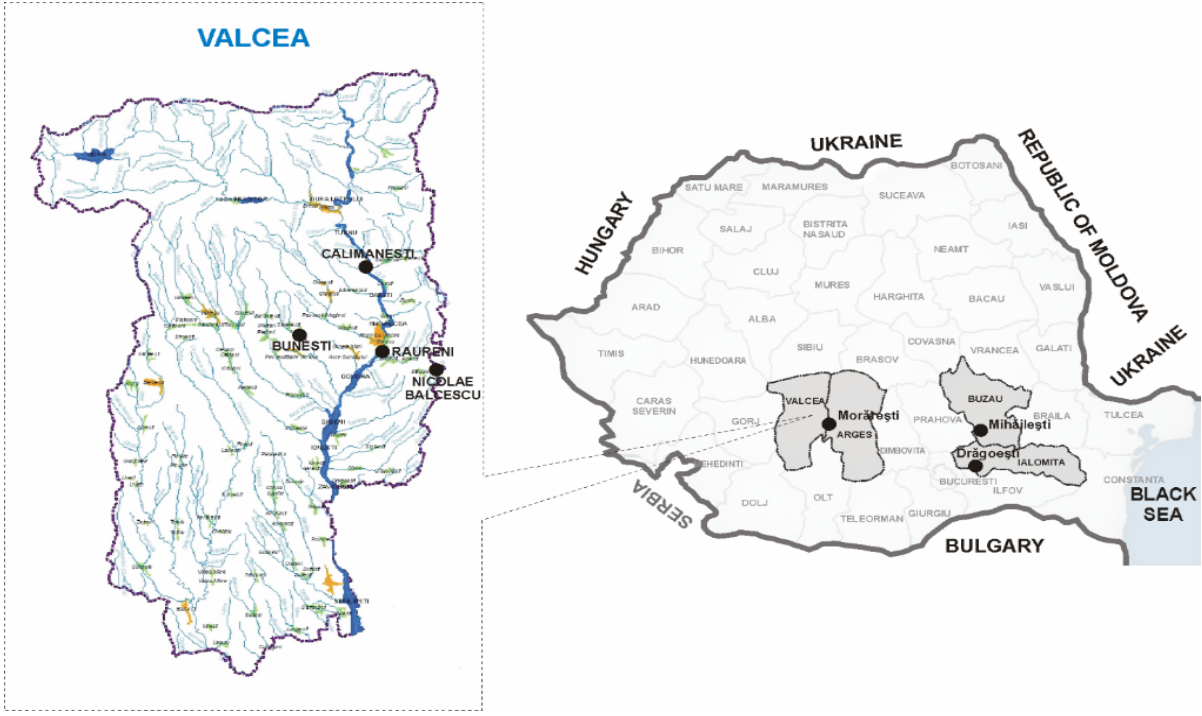

Figure 4. Location of sampling sites and surrounding areas

Table 3. Sampling sites

\begin{tabular}{|c|c|c|c|c|c|c|}
\hline \multirow{2}{*}{ County } & $\begin{array}{c}\text { Location of } \\
\text { farm/milk } \\
\text { collecting } \\
\text { centre }\end{array}$ & $\begin{array}{c}\text { Site } \\
\text { code }\end{array}$ & $\begin{array}{c}\text { Altitude } \\
\text { (average) }\end{array}$ & Latitude & Longitude & Description \\
\cline { 2 - 7 } & Calimanesti & VL-N & $288 \mathrm{~m}$ & $45.23910 \mathrm{~N}$ & $24.33986 \mathrm{E}$ & $\begin{array}{c}\text { Resort/near } \\
\text { mountain area }\end{array}$ \\
\cline { 2 - 7 } & Bunesti & VL-W & $303 \mathrm{~m}$ & $45.11434 \mathrm{~N}$ & $24.18899 \mathrm{E}$ & $\begin{array}{c}\text { Rural/Agricultural } \\
\text { area }\end{array}$ \\
\cline { 2 - 7 } & Raureni & VL-S & $227 \mathrm{~m}$ & $45.06129 \mathrm{~N}$ & $24.33649 \mathrm{E}$ & $\begin{array}{c}\text { Urban/near } \\
\text { industrial area }\end{array}$ \\
\cline { 2 - 7 } & $\begin{array}{c}\text { Nicolae } \\
\text { Balcescu }\end{array}$ & VL-E & $490 \mathrm{~m}$ & $45.10656 \mathrm{~N}$ & $24.43600 \mathrm{E}$ & $\begin{array}{c}\text { Rural/Agricultural } \\
\text { area }\end{array}$ \\
\hline Arges & Moraresti & AG & $548 \mathrm{~m}$ & $45.01610 \mathrm{~N}$ & $24.56791 \mathrm{E}$ & $\begin{array}{c}\text { Rural/Agricultural } \\
\text { area }\end{array}$ \\
\hline lalomita & Dragoesti & $\mathrm{IL}$ & $66 \mathrm{~m}$ & $44.56535 \mathrm{~N}$ & $26.54118 \mathrm{E}$ & $\begin{array}{c}\text { Rural/Agricultural } \\
\text { area }\end{array}$ \\
\hline Buzau & Mihailesti & $\mathrm{BZ}$ & $75 \mathrm{~m}$ & $44.91444 \mathrm{~N}$ & $26.67677 \mathrm{E}$ & $\begin{array}{c}\text { Rural/Agricultural } \\
\text { area }\end{array}$ \\
\hline
\end{tabular}


DIANA IONELA STEGARUS, ROXANA ELENA IONETE,

SIMONA OANCEA, ECATERINA LENGYEL, ADINA FRUM

\section{Reagents and reference solutions}

Mix standard solutions for organochlorine pesticides $(\alpha-\mathrm{HCH}, \beta-\mathrm{HCH}$, $\delta-\mathrm{HCH}$, aldrin, dieldrin, endrin, DDE, DDD, and DDT) and $\mathrm{PCB}$ congeners (PCB28, PCB52, PCB101, PCB138, PCB153, PCB180, and PCB194) were purchased from LGC (Germany). Proper amounts of stock solutions of all these standards were prepared in chromatography-grade $n$-hexane. Working standard solutions were prepared by appropriate dilution of the above mix standards in $n$-hexane at different concentrations for both, pesticides and PCBs. For each compound, linearity at six different concentrations, of 0.100 , $0.070,0.050,0.010,0.005$, and $0.001 \mu \mathrm{g} / \mathrm{L}$, with triplicate measurements, were evaluated through a linear regression analysis. Both stock and working standards were stored at $-24^{\circ} \mathrm{C}$ during the study.

Chromatography-grade acetonitrile, acetone, ethyl acetate, $n$-hexane, dichloromethane and sodium chloride were obtained from Merck (Darmstadt, Germany); anhydrous sodium sulphate of analytical grade was produced by Sigma Aldrich (Germany).

A multi-element standard solution XVI CertiPUR (a mixture of 21 elements in diluted nitric acid), with a certified value of $100 \pm 3 \mathrm{mg} / \mathrm{L}$, purchased from Merck (Darmstadt, Germany) was used for the calibration curve in the quantitative analysis of metals $(\mathrm{Pb}, \mathrm{Cd}, \mathrm{Cu}$, and $\mathrm{Zn})$. Solutions of varying concentrations were prepared for the metals by diluting the standard solution. $65 \% \mathrm{HNO}_{3}$ from Merck (Darmstadt, Germany) and ultrapure water, with a maximum resistivity of $18.2 \mathrm{M} \Omega / \mathrm{cm}$, produced by a Milli-Q Millipore water purification system (Bedford, MA, USA), were used for sample treatment and dilution.

\section{Extraction}

Organochlorine pesticides from water and bovine milk - $10 \mathrm{~mL}$ of sample were introduced into a $100 \mathrm{~mL}$ centrifugation tube, $5 \mathrm{~g}$ natrium chloride $(\mathrm{NaCl})$ and $30 \mathrm{~mL}$ of acetonitrile were added. The tubes were stirred at $250 \mathrm{rpm}$ for 20 minutes, using an AREX Heating Magnetic Stirrer from VELP (Italy). The mixtures were centrifuged at $6000 \mathrm{rpm}$ for 15 minutes for obtaining clear sample layers. After the centrifugation, approximately $90 \%$ of the acetonitrile superior layer was transferred into a centrifugation tube. Then, we added $5 \mathrm{~g}$ of anhydrous sodium sulphate and stirred the tube for 2 minutes. The sample was kept into the freezer for 15 minutes; next, $10 \mathrm{~mL}$ of the acetonitrile superior layer was mixed with $10 \mathrm{~mL}$ of a saturated solution of sodium chloride and $30 \mathrm{~mL}$ of ultrapure water, and then extracted twice with $20 \mathrm{~mL}$ dichloromethane using a separation funnel. The organic layer was filtered by using sodium sulphate and then evaporated to dryness. 
The residue was taken with $2 \mathrm{~mL}$ of ethyl acetate and filtered by using filter paper purchased from Sigma Aldrich. The obtained solution was transferred into a $1.700 \mathrm{~mL}$ flask, analyzed and quantified by using the gas chromatography with an electron capture detector (GC/ECD), the results being confirmed after by using gas chromatography coupled with mass spectrometry (GC/MS).

Organochlorine pesticides and PCBs from soil and grass - $20 \mathrm{~g}$ of cryogenically triturated samples were stirred intensively for 15-20 minutes with $50 \mathrm{~mL}$ of acetone using an AREX Heating Magnetic Stirrer from VELP (Italy). $50 \mathrm{~mL}$ of ether were added, and the stirring continued for another 15 minutes. This procedure was executed twice. The combined extracts were washed twice into a separation funnel by using $500 \mathrm{~mL}$ distilled water. The resulted extract was dried on sodium sulphate (Sigma Aldrich, Germany) and concentrated at $1 \mathrm{~mL}$, after which the sodium sulphate was washed three times with $10 \mathrm{~mL}$ of ether. In order to obtain satisfactory results, the acetone water ratio was 9:1, and the acetone ether ratio was kept constantly at 1:2.

Sample purification - Into a glass column of $25 \mathrm{~cm}$ length and $1 \mathrm{~cm}$ interior diameter, designed with a tap, a quartz wool plug and $3 \mathrm{~g}$ of aluminum oxide were inserted. The concentrate extract was introduced into the dry column by using a pipette. The concentration flask was washed twice with $1 \mathrm{~mL}$ of ether and the washing portions were transferred into the column immediately after the liquid reached the superior part of the columns filling. The elution was performed by using $20 \mathrm{~mL}$ ether and the resulted extract was concentrated to $1 \mathrm{~mL}$. To the final extract, $1 \mu \mathrm{L}$ of reference solution for the injection was added.

$P C B$ s from water and bovine milk - The extraction was performed by using $500 \mathrm{~mL}$ of sample and $15 \mathrm{~mL}$ of $\mathrm{n}$-hexane stirred for 1 hour. The organic layer was dried with sodium sulphate and concentrated to $2 \mathrm{~mL}$ by using a Turbo Vap 500 concentrator. The $2 \mathrm{~mL}$ were passed through a glass column designed with a tap and a glass wool plug, filled with silica gel 60 (70-230 mesh), with $7 \mathrm{~cm}$ length. The second layer was made of $0.5 \mathrm{~cm}$, silica gel 60 (20 mesh). The column was washed with $10 \mathrm{~mL} n$-hexane, after which the samples was run through and the column was washed again with $5 \mathrm{~mL} \mathrm{n}$-hexane. The extract was concentrated to $1 \mathrm{~mL}$ and then injected into the apparatus.

Heavy metals - Into a reaction vessel $1 \mathrm{~g}$ of dried sample (soil or grass) and $10 \mathrm{~mL}$ of $\mathrm{HCl}: \mathrm{HNO}_{3}(3: 1)$ solution were inserted and left to react for 10 minutes, and then put through the digestion process [31]. $30 \mathrm{~mL}$ of milk were dried and solubilized in $35 \mathrm{~mL}$ of $\mathrm{HNO}_{3} 0.3 \%$ solution and then put through the digestion process [32]. For the determination of element contents, samples were digested with a microwave oven, at $400 \mathrm{~W}, 800 \mathrm{PSI}$ pressure and a temperature of $180^{\circ} \mathrm{C}$, maintained for 20 minutes. 
DIANA IONELA STEGARUS, ROXANA ELENA IONETE, SIMONA OANCEA, ECATERINA LENGYEL, ADINA FRUM

\section{Chromatographic analysis of PCBs and pesticides}

A number of 7 PCB congeners (PCB28, PCB52, PCB101, PCB138, PCB153, PCB180, PCB194) and 10 organochlorine pesticides $(\alpha-, \beta-, \delta-\mathrm{HCH}$, aldrin, dieldrin, endrin, DDE, DDD, DDT and endosulfan) were measured by a GC-MS apparatus (Varian 450-GC coupled with a Varian 240-MS external ionization ion trap MS) in the collected samples (soil, water, grass and milk). Helium (purity 99.9999\%) was employed as the carrier gas at a flow rate of $1 \mathrm{~mL} / \mathrm{min}$. Chromatographic separation was performed using a CP-Sil $8 \mathrm{CB}$ column $(50 \mathrm{~m} \times 0.25 \mathrm{~mm} \times 0.25 \mu \mathrm{m}$, Agilent). During the analysis, the injector and detector temperatures were maintained at $240^{\circ} \mathrm{C}$ and $270{ }^{\circ} \mathrm{C}$. The temperature of the mass spectrometer ion source was maintained at $210^{\circ} \mathrm{C}$. The oven temperature was initially set at $60^{\circ} \mathrm{C}$ (maintained for 5 minutes) and continued by increasing to $280^{\circ} \mathrm{C}$ (at a rate of $20^{\circ} \mathrm{C} / \mathrm{min}$ ). An amount of $2 \mu \mathrm{L}$ from each sample (extracts, standards and blanks) was used for injection in the apparatus.

The determination of the recovery yield - For every batch of analyzes performed, a sample of contaminants was prepared by using known concentrations from the compounds of interest. The analytical method was validated on the working matrix. The samples were divided in quantities of $20 \mathrm{~g}$ each and treated with a pesticide mix of concentrations between 0.05 and $10 \mu \mathrm{g} / \mathrm{L}$. For the pesticide mix recovery tests, six replicas of every concentration were determined for the analyzed soil. The calibration curve and the samples' matrix were determined in double at the concentration levels stated before.

\section{Heavy metals analysis}

Concentrations of lead, cadmium, copper and zinc were measured by a method proposed by Miedico et al. in 2017 [33]. The quantification was performed by using calibration curves, determined with analytical standards purchased from LGC Dr. Ehrenstorfer.

\section{Statistical analysis}

Data are presented as a result of three replicas. Mean, standard deviation, maximum and minimum ranges were calculated using simple statistical analysis, and the correlation of variables was done by calculation of Pearson's correlation coefficients at significance level of risk $\alpha \leq 5 \%$.

\section{ACKNOWLEDGMENTS}

This work is supported by the project ANTREPRENORDOC, in the framework of Human Resources Development Operational Programme 20142020, financed from the European Social Fund under the contract number 36355/23.05.2019 HRD OP /380/6/13 - SMIS Code: 123847. 
THE IMPACT OF ANTHROPOGENIC ACTIVITIES TO THE LEVEL OF SOME POPS RESIDUES IN BOVINE MILK FROM SEVERAL FARMS IN ROMANIA

This work is part of the project PN 19110303 "Advanced techniques for identifying sources of contamination and biochemical reactions in aquatic ecosystems" financed by the Romanian Ministry of Research and Innovation.

\section{REFERENCES}

[1]. R. Jayaraj; P. Megha; P. Sreedev; Int. Toxicol., 2016, 9(3-4), 90-100

[2]. Y. Liu; S. Li; Z. Ni; M. Qu; D. Zhong; C. Ye; F. Tang; Sci. Total Environ., 2016, 542(15), 620-628

[3]. M.G. Barron; Z.J. Ashurova; M.A. Kukaniev; H.K. Avloev; K.K. Khaidarov; J.N. Jamshedov; O.S. Rahmatullova; S.S. Atolikshoeva; S.S. Mamadshova; O. Manzenyuk; Environ. Pollut., 2017, 224, 494-502

[4]. K. Ki-Hyun; K. Ehsanul; A.J. Shamin;, Sci. Total Environ., 2017, 575, 525-535

[5]. O.M.L. Alharbi; A.A. Basheer; R.A. Khattab; I. Ali; J. Mol. Liq., 2018, 263, 442-453

[6]. FAO and WHO. Codex Alimentarius, International food standards, adopted in 2006, revised in 2018, CXC 62-2006

[7]. O.M. Faroon; L.S. Keith; C. Smith Simon; C.T. De Rosa; WHO, 2003

[8]. S. Oancea, The impact of persistent organic pollutants on human health, in The impact of persistent organic pollutants on freshwater ecosystems and human health, A. Curtean-Banaduc Ed.; "Lucian Blaga" University of Sibiu Publisher, Sibiu, 2016, pp. 57-84

[9]. X. Chen; Y. Yin; K. Dang; B. Puschner; PLoS ONE, 2017, 12(1): e0170129

[10]. F. Donato; M. Magoni; R. Bergonzi; C. Scarcella; A. Indelicato; S. Carasi; P. Apostoli; Chemosphere, 2006, 64(9), 1562-1572

[11]. M.M. Storelli; C. Scarano; C. Spanu; E.P.L. De Santis; V.P. Busco; A. Storelli; G.O. Marcotrigiano; Food Chem. Toxicol., 2012, 50, 1413-1417

[12]. A. Abballe; P.G. Barbieri; A. Di Domenico; S. Garattini; N. lacovella; A.M. Ingelido; V. Marra; R. Miniero; S. Valentini; E. Felip, Chemosphere, 2013, 90, 49-56

[13]. P. Tremolada; N. Guazzoni; M. Parolini; B. Rossaro; M.M. Bignazzi; A. Binelli; Sci. Total Environ., 2014, 487, 471-480

[14]. L. Bertocchi; S. Ghidini; G. Fedrizzi; V. Lorenzi; Environ. Sci. Pollut. R., 2015, 22(13), 9775-9785

[15]. V. Page; U. Feller; Agron., 2015, 5, 447-463

[16]. L. Levei; E. Kovacs; M.A. Hoaghia; A. Ozunu; Studia UBB Chemia, 2018, 1, 87-98

[17]. R.M. Hategan; G.M. Popita; I. Varga; A. Popovici, T. Frentiu; Studia UBB Chemia, 2012, 1, 119-126

[18]. V.D.Dimitrijevic; M.N. Stankovic; D.M. Dordevic; I.M. Krstic; M.G. Nikolic; A.I.j. Bojic; N.S. Krstic; Studia UBB Chemia, 2019, 1, 19-39

[19]. M. Jaishankar; T. Tseten; N. Anbalagan; B.B. Mathew; K.N. Beeregowda; Int. Toxicol., 2014, 7(2), 60-72 


\section{DIANA IONELA STEGARUS, ROXANA ELENA IONETE, SIMONA OANCEA, ECATERINA LENGYEL, ADINA FRUM}

[20]. K. Rehman; F. Fatima; I. Waheed; M.S.H. Akash; J. Cell. Biochem., 2018, 119(1), 157-184

[21]. D.M. Popa; M. Moldovan; D. Prodan; C. Aldea; C. Lazar; C. Lazea; M. Crisan; L.M. Sur; S.C. Man; C. Borzan; Environ. Geochem. Health., 2020, DOI:10.1007/ s10653-020-00750-9

[22]. F. Miculescu; M. Miculescu; L.T. Ciocan; A. Ernuteanu; I. Antoniac; I. Pencea; E. Matei; Dig. J. Nanomater. Bios., 2011, 6(3), 1117-1127

[23]. Ministry of Water, Forests and Environmental Protection, Order no. 756/1997, Romanian Official Monitor, 1997, revised in 2011

[24]. Directive 2002/32/EC of the European Parliament and of the Council of 7 May 2002, Official Journal of the European Union, 2002, L140, 10-21

[25]. Commission Regulation (EC) No 1259/2011 of 2 December, Official Journal of the European Union, 2011, L320, 18-23

[26]. Commission Regulation (UE) nr. 574/2011 of 16 June 2011, Official Journal of the European Union, 2011, L159, 7-24

[27]. Romanian Parliament, Law no. 311/2004, Romanian Official Monitor, 2004

[28]. National Sanitary, Veterinary and Food Safety Authority, Order no. 23/2007, Romanian Official Monitor, 2007

[29]. H. Deti; A. Hymete; A.A. Bekhit; A.M.I. Mohamed; A.E.D.A. Bekhit; Chemosphere, 2014, 106, 70-74

[30]. Romanian Ministry of the Environment and Water Management, Order no. 344/ 2004, Romanian Official Monitor, 2004

[31]. L. Yun; K.B. Jensen; Larson. J. Agr. Sci.Bot., 2018, 2(1), 19- 24

[32]. A. Ayar; D. Sert; N. Akin; Environ. Monit. Assess., 2009, 152, 1-12

[33]. O. Miedico; M. Iammarino; M. Tarallo; A.E. Chiaravalle; Int. J. Food Prop., 2017, 20(12), 2888-2900 